\title{
Multimodality imaging in amyloidosis
}

\author{
Stephanie Howard ${ }^{a}$, Jyothi Jagannathan ${ }^{a}$, Katherine Krajewski ${ }^{a}$, Angela Giardino ${ }^{a}$, \\ Katherine Zukotynski ${ }^{a}$, Kevin O’Regan ${ }^{b}$, Irene Ghobrial ${ }^{a}$, Nikhil Ramaiya ${ }^{a}$ \\ ${ }^{a}$ Department of Radiology, Dana Farber Cancer Institute, 450 Brookline Avenue, Boston, MA 02115, USA; \\ ${ }^{b}$ Department of Radiology, University of Cork, Cork, Ireland \\ Corresponding address: Stephanie Anne Holler Howard, MD, Department of Cancer Imaging/Radiology, \\ Dana Farber Cancer Institute, Harvard Medical School 450 Brookline Ave, Boston, MA 02114, USA. \\ Email: sahoward@partners.org
}

Date accepted for publication 5 March 2012

\begin{abstract}
Amyloidosis comprises a rare spectrum of protein deposition diseases that diffusely or focally affect any organ. Amyloid's variable clinical presentation and nonspecific disease course often cause it to evade early diagnosis. This pictorial essay aims to familiarize radiologists with the pathophysiology of amyloidosis, to describe the basic classifications of amyloidosis, and to use multimodality imaging to illustrate its varied appearance throughout the body. This review highlights the diagnostic challenge of interpreting radiographic studies in patients with hematologic malignancies and concurrent amyloidosis. Radiologists should consider amyloid in chronically ill patients or patients with hematologic malignancies who have unusual/unexpected imaging findings.
\end{abstract}

Keywords: Amyloid; amyloidosis; multiple myeloma.

\section{Introduction}

Amyloid deposition typically occurs systemically, diffusely infiltrating organs. However $10-20 \%$ of cases present as focal amyloidomas ${ }^{[1-3]}$. The type of protein deposited determines the subgroup of amyloid. In primary amyloidosis, the most common subtype, a fragment of the immunoglobulin light chain is deposited ${ }^{[2]}$. Hence, primary amyloidosis is associated with plasma cell dyscrasias, namely multiple myeloma and Waldenström macroglobulinemia/lymphoplasmacytic lymphoma ${ }^{[2,4]}$. In secondary amyloidosis, the protein deposited is produced in response to inflammation; hence secondary amyloidosis occurs in chronic inflammatory conditions such as rheumatoid arthritis or tuberculosis ${ }^{[1]}$. A third, hereditary or senile systemic type of amyloid (ATTR) is derived from mutant and wild type transthyretin $(\mathrm{TTR})^{[1]}$.

Diagnosis of amyloidosis is confirmed by the presence of apple-green birefringence on polarized light examination of a tissue biopsy stained with Congo Red ${ }^{[1]}$. Typing is usually based on clinical and laboratory findings with monoclonal gammopathy evaluation and, if indicated, TTR gene testing. Among patients with multiple myeloma, 10\% have evidence of concurrent amyloido$\operatorname{sis}^{[2]}$. Serum and urine immunofixation studies reveal an $\mathrm{M}$ protein in more than $80 \%$ of patients, with light chains more frequent than heavy chains and with lambda light chain more common than kappa light chain ${ }^{[2]}$. The main symptoms and signs related to amyloidosis include unexplained fatigue or weight loss, cardiomyopathy, orthostatic hypotension, macroglossia, nephrotic syndrome, carpal tunnel syndrome, peripheral or autonomic neuropathy, periorbital purpura, or hepatomegaly ${ }^{[1,2]}$. Survival is usually poor, and shortest in those patients with cardiomyopathy ${ }^{[2]}$. Treatment of amyloidosis is similar to that for multiple myeloma and includes melphalan and dexamethasone, autologous stem cell transplantation, and novel therapies including bortezomib, lenalidomide, and pomalidomide ${ }^{[2]}$.

Primary amyloidosis commonly involves the kidneys or heart, often presenting with edema in the setting of nephrotic syndrome or congestive heart failure ${ }^{[1]}$. Median survival in primary amyloidosis is 1.5 years, versus 4.5 years in secondary amyloidosis, which tends to involve the abdomen, and rarely the heart ${ }^{[1,3]}$. ATTR (hereditary) amyloid may involve the heart, but amyloidosis progresses more quickly, making a timely diagnosis

This paper is available online at http://www.cancerimaging.org. In the event of a change in the URL address, please use the DOI provided to locate the paper. 
critical $^{[1]}$. Amyloidosis causes more organ disease than ATTR, which often presents with peripheral sensorimotor and autonomic neuropathy ${ }^{[1]}$. Increased incidence of amyloidosis is in part due to longer survival of patients with chronic inflammatory diseases ${ }^{[4]}$. This article uses multimodality imaging to review the appearance of primary and secondary amyloidosis throughout the body.

\section{Brain}

Intracerebral amyloid (also known as amyloid angiopathy) is not usually associated with systemic amyloidosis, and is characterized by protein deposition in the media and adventitia of small to medium sized cortical and leptomeningeal vessels, leading to vessel damage, hemorrhage, and infarction ${ }^{[5-7]}$. Chronic hypoperfusion leads to leukoencephalopathy and atrophy, manifesting as periventricular hypodensity on computed tomography (CT), or hyperintensity on T2-weighted images on magnetic resonance imaging $(\mathrm{MRI})^{[6]}$. Recurrent subcortical and cortical hemorrhages sparing the deep white matter are typical, with susceptibility imaging raising diagnostic sensitivity by facilitating microhemorrhage detection ${ }^{[6,7]}$. At autopsy, amyloid deposition affects $33 \%$ of $60-70$ year olds, and $>75 \%$ of individuals over 90 years $^{[7]}$. Fig. 1 illustrates the appearance of intracerebral amyloidosis.

\section{Head and neck}

Amyloid may occur anywhere in the neck, although laryngeal involvement is most common ${ }^{[5]}$. CT of the head and neck reveals diffuse or focal organ infiltration, with or without calcification ${ }^{[5]}$. Involvement of cervical lymph nodes is rare, even in systemic amyloidosis, with nodes often appearing with intermediate intensity on T1-weighted images, and decreased intensity on T2-weighted images ${ }^{[8]}$. Extranodal amyloid in the head and neck tends to maintain an iso- to hypointense appearance on T1-weighted imaging, and may be hypo, iso, or minimally hyperintense on T2-weighted imaging ${ }^{[5]}$. Fig. 2 illustrates nasopharyngeal amyloidosis. Intraorbital amyloid (Fig. 3) often involves the lacrimal gland ${ }^{[5]}$.

\section{Pulmonary}

Fifty percent of amyloid patients have respiratory involvement, with most clinically significant cases occurring in patients with primary amyloidosis ${ }^{[9]}$. The 4 main patterns of pulmonary involvement are lymphatic, diffuse alveolar septal, nodular parenchymal, and tracheobronchial, with the former 2 patterns seen more commonly in patients with systemic involvement, and the latter 2 patterns seen more commonly with localized disease ${ }^{[9]}$.

Lymphatic involvement may be bulky and calcified, occurring in up to $75 \%$ of patients with primary amyloi$\operatorname{dosis}^{[9]}$. Diffuse alveolar septal amyloid is due to interstitial/small vessel involvement, with CT revealing reticular/ ground glass opacities and interlobular septal thickening, often with adjacent 2-4 mm subpleural micronodules ${ }^{[9]}$. Nodular parenchymal amyloid presents with single or multiple pulmonary nodules that are usually peripheral, well-defined, and sharply marginated, with 30-50\% of cases exhibiting calcification ${ }^{[9,10]}$. The nodules grow slowly, without regression, and may cavitate ${ }^{[3,10]}$.

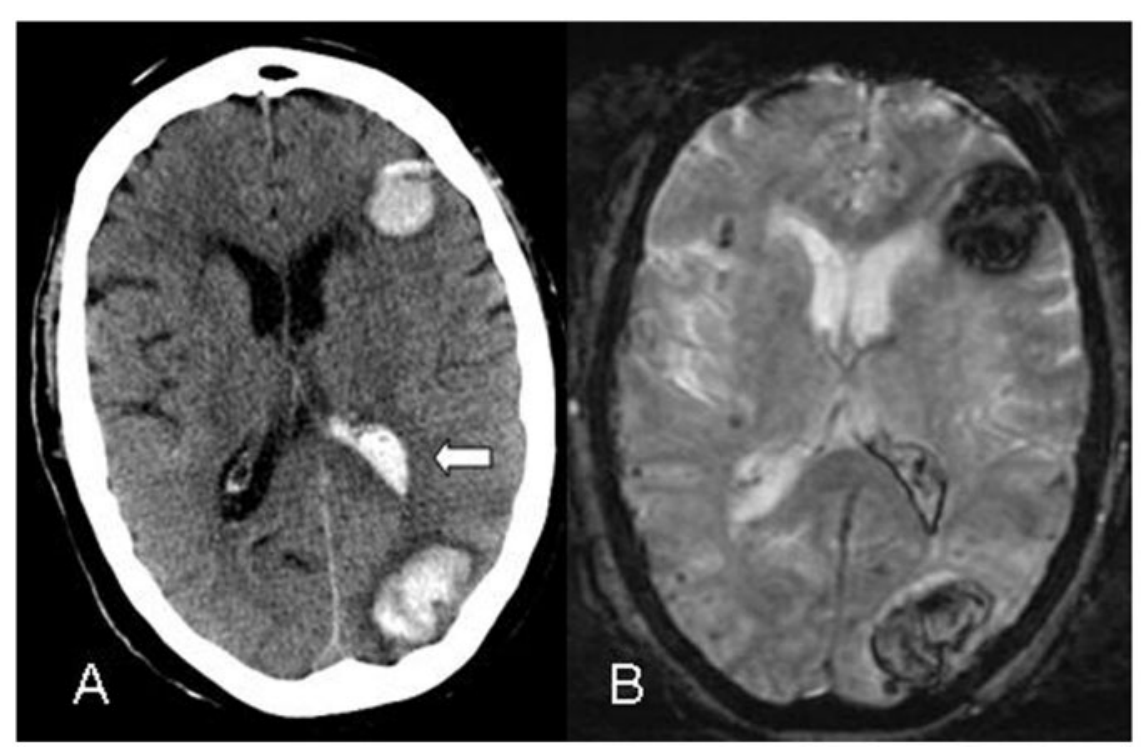

Figure 1 A 75-year-old man presenting with confusion. (A) Non-contrast axial CT image at the level of the lateral ventricles reveals large subcortical hyperdense lesions in the frontal and parietal lobes; the hyperdensity suggests hemorrhage. Hemorrhage layers in the posterior horn of the left lateral ventricle (arrow). (B) Axial T1-weighted gradient echo MR sequence confirms intralesional and intraventricular hemorrhage. 


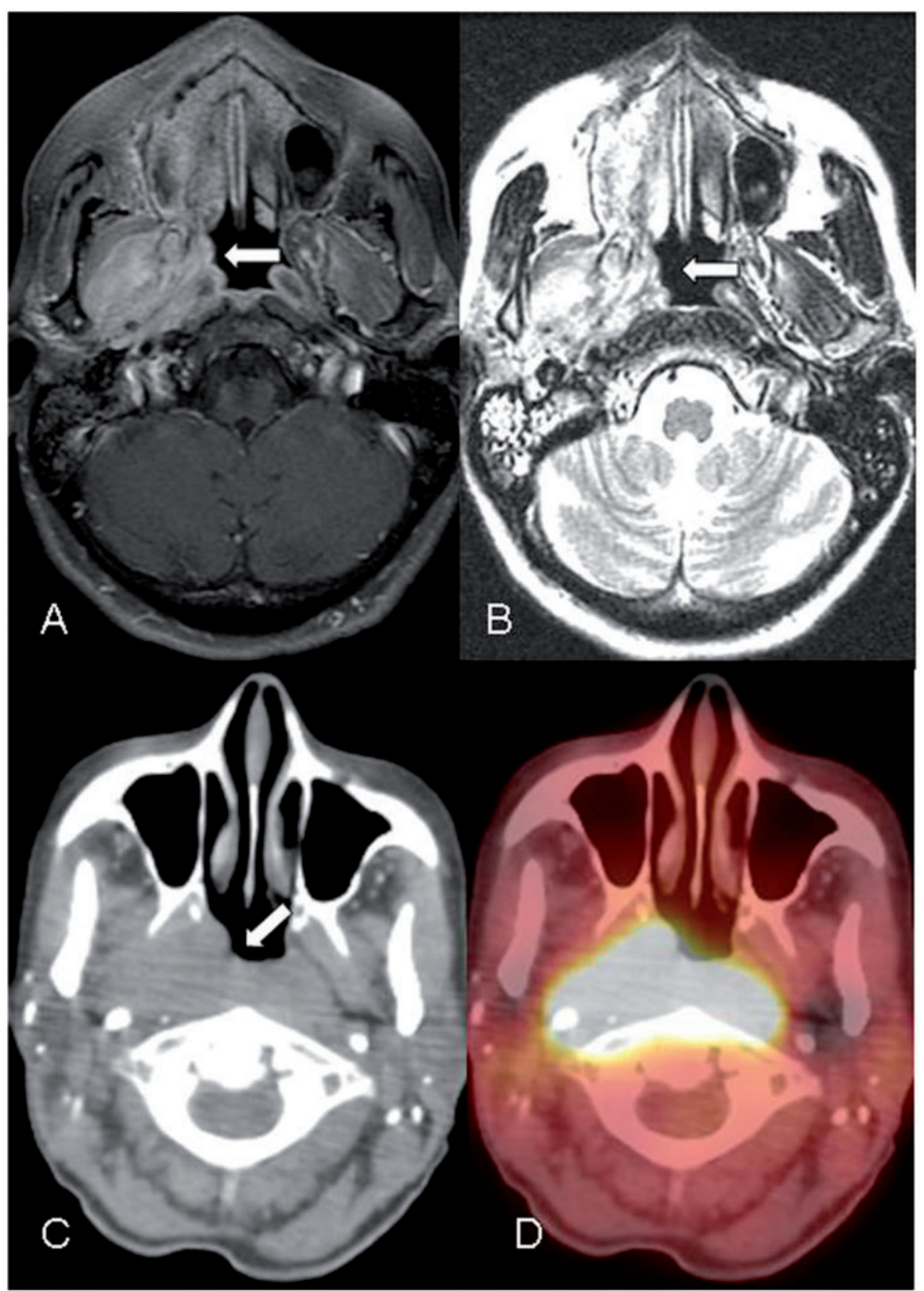

Figure 2 A 62-year-old with a history of lymphoplasmacytic lymphoma. (A) Axial T1-weighted postcontrast image reveals a large, avidly enhancing mass in the right parapharyngeal space involving the medial and lateral ptygeroid muscles and partially encasing the right internal carotid artery. (B) T2-weighted image at the same level reveals a mildly heterogeneous, predominantly hyperintense mass. (C) Axial unenhanced CT image obtained just inferiorly reveals extension of this mass across the midline. (D) Fused axial PET/CT image reveals an intensely FDG avid mass involving the right parapharyngeal space and extending across the midline (standardized uptake value (SUV) $)_{\max } 20.0$ ). Complete surgical resection of this mass revealed diffuse amyloidosis, with no lymphomatous involvement.

Tracheobronchial amyloid manifests as focal or diffuse discontinuous submucosal deposits, which are often calcified $^{[9]}$.

Pulmonary amyloid often presents clinically with recurrent pneumonia, likely due to interstitial involvement in diffuse alveolar septal amyloid, or obstruction in the setting of lymphatic or tracheobronchial amyloid ${ }^{[4]}$. Average survival in patients with systemic amyloidosis and pulmonary involvement is 16 months ${ }^{[10]}$. Figs, 4, 5 and 6 show patients with lymphatic, diffuse alveolar septal, and nodular parenchymal amyloid.

\section{Cardiac}

Primary amyloidosis frequently involves the heart, and average survival in patients with detectable troponin 


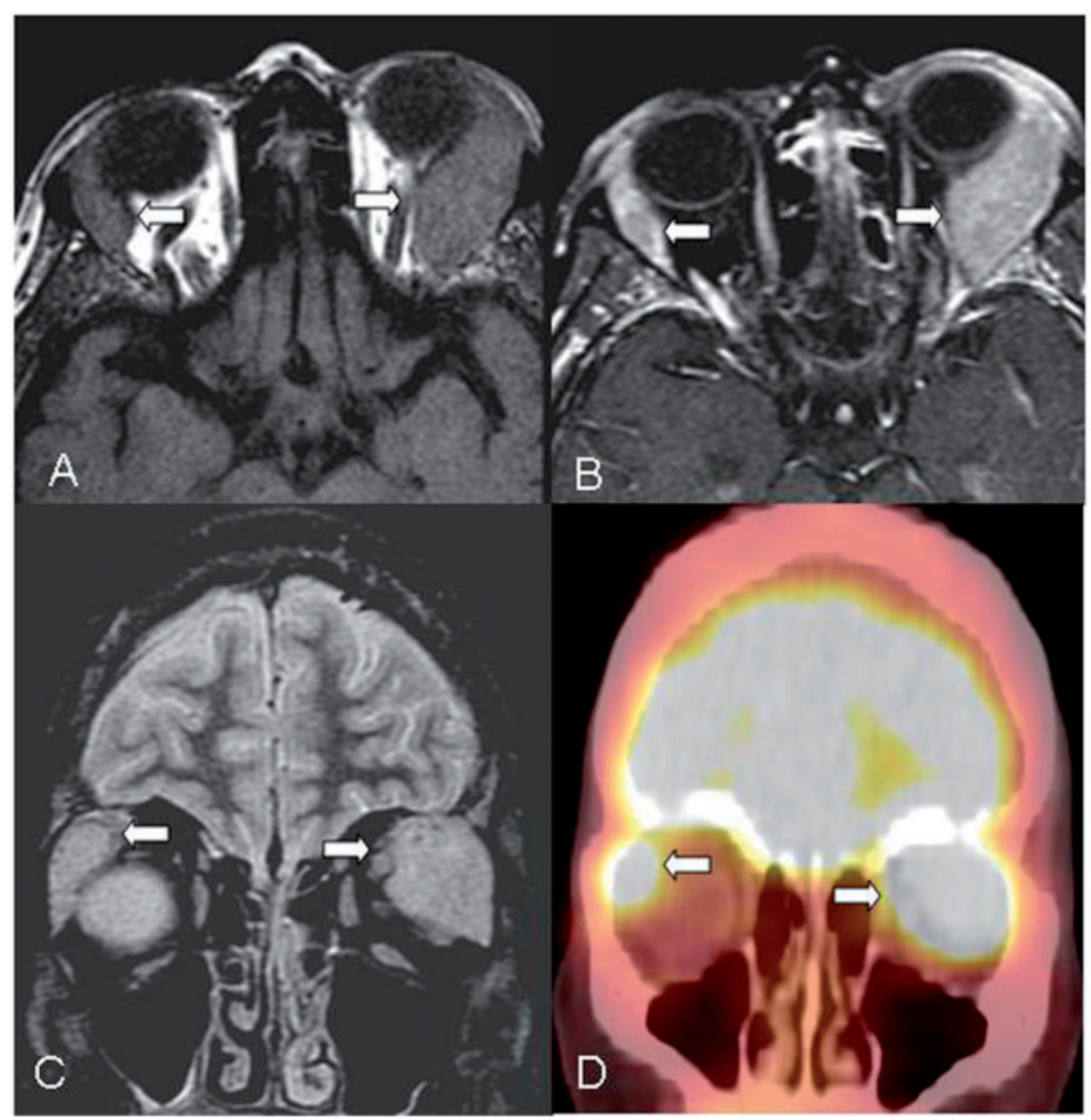

Figure 3 A 60-year-old with a history of lymphplasmacytic lymphoma and new onset double vision. Axial pre- (A) and post-contrast (B) T1-weighted MR images show hypointense enhancing masses (arrows), involving bilateral lacrimal glands. (C) Coronal short tau inversion recovery (STIR) image shows hyperintense lacrimal masses bilaterally. (D) Coronal fused image from an FDG-PET/CT shows intense FDG avidity in bilateral masses $\left(\mathrm{SUV}_{\max } \mathbf{3 2 . 3}\right.$ left side, 17.5 right side). Biopsy of bilateral lacrimal glands confirmed lymphomatous involvement, intermixed with amyloid deposition.

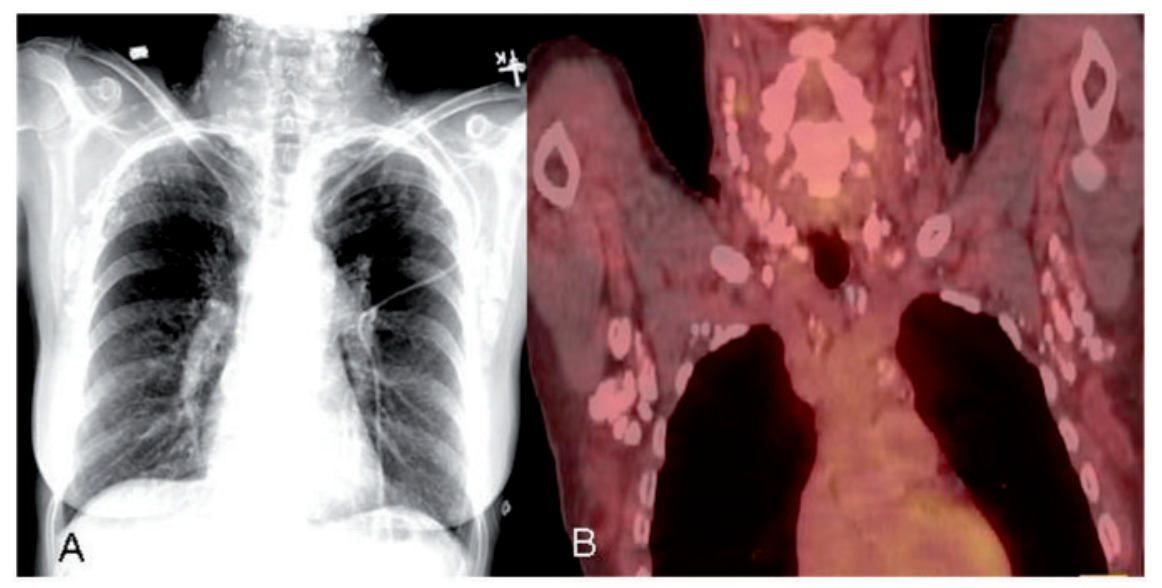

Figure 4 A 60-year-old woman with a history of Waldenström macroglobulinemia/lymphoplasmacytic lymphoma presenting with neck swelling. (A) Chest radiograph reveals extensive calcified cervical, supraclavicular, axillary, and mediastinal lymphadenopathy. (B) Fused coronal images from a PET/CT shows little FDG avidity in the calcified lymphadenopathy (right axilla $S U V_{\max }$ 1.2). Biopsy of the right axilla confirmed primary amyloid infiltration, with minimal evidence of lymphoma. 


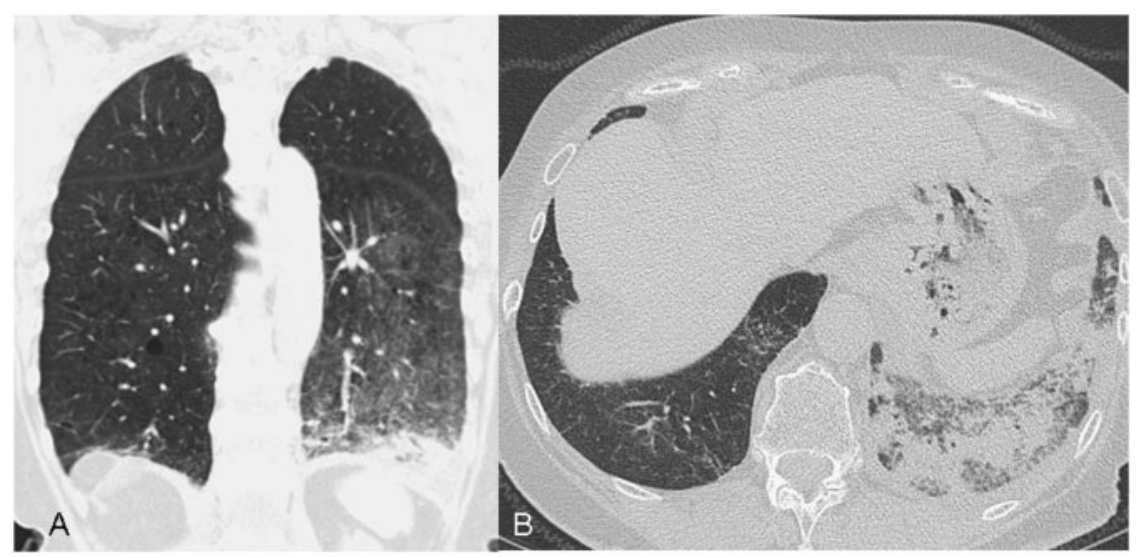

Figure 5 An 80-year-old woman with no significant past medical history presenting with recurrent pneumonias/hemoptysis. Coronal (A) and high-resolution axial (B) CT images performed without intravenous contrast reveal a consolidative opacity in the basilar segments of the left lower lobe superimposed on a background of ground glass opacity and smooth interlobular septal thickening, consistent with pneumonia superimposed on diffuse alveolar septal amyloid. Left lower lobe transbronchial biopsy revealed pulmonary amyloidosis.

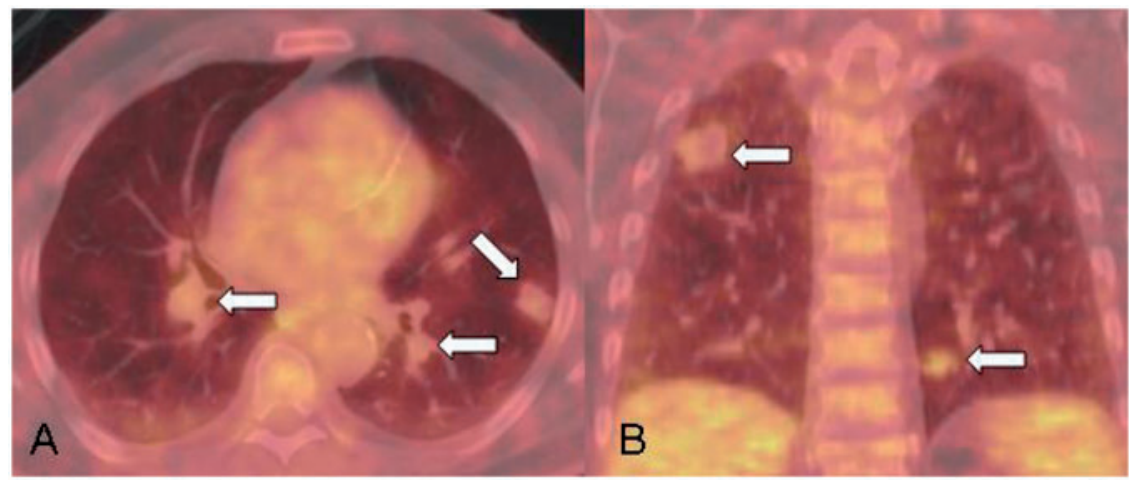

Figure 6 A 68-year-old man with no significant past medical history presenting with a cough. Fused axial images from a PET/CT confirm multiple pulmonary nodules with little to no FDG avidity above background (SUV max $_{\text {in nodules ranged }}$ from 1.5 to 2.7). Surgical resection of a left upper lobe nodule revealed amyloid, with no evidence of malignancy.

levels is only 6 months, versus 22 months in troponinnegative patients ${ }^{[2]}$. Although signs of restrictive cardiomyopathy raise the possibility of cardiac amyloid on echocardiography and CT, cardiac MRI is increasingly utilized in myocardial evaluation. On MRI, cardiac amyloid often manifests as concentric biventricular myocardial hypertrophy with dilated atria and non-dilated ventricles, thickening of the intra-atrial septum, and diffuse subendocardial delayed enhancement in a nonvascular distribution ${ }^{[1]}$. The ejection fraction is usually maintained until disease becomes advanced. Pericardial and pleural effusions are common in advanced disease $^{[11]}$. Figs. 7 and 8 demonstrate cardiac amyloidosis.

\section{Abdominal}

The gastrointestinal tract is involved in up to $98 \%$ of patients with systemic amyloidosis ${ }^{[12]}$. Protein deposition occurs mainly around arterioles and muscles, leading to ischemia and dysmotility ${ }^{[12]}$. Fluoroscopic and CT imaging of the gastrointestinal tract is characterized by focal or diffuse wall thickening that is often symmetric and due to edema in the setting of ischemia caused by vascular deposition $^{[12]}$. Primary amyloidosis more commonly presents with polypoid protrusions and fold thickening, while secondary amyloidosis often results in a coarse mucosal pattern with innumerable fine granular nodules ${ }^{[12]}$. The prevalence of histological and radiological abnormalities is highest in the small bowel ${ }^{[12]}$. Although $50 \%$ of patients with systemic amyloidosis have gastric involvement, these patients rarely have clinical symptoms. The distal colon is commonly involved at autopsy, however these lesions are often radiographically occult ${ }^{[12]}$. Fig. 9 shows a case of amyloid involving the small bowel.

Solid visceral involvement may occur focally or diffusely, often causing decreased attenuation on CT, with or 


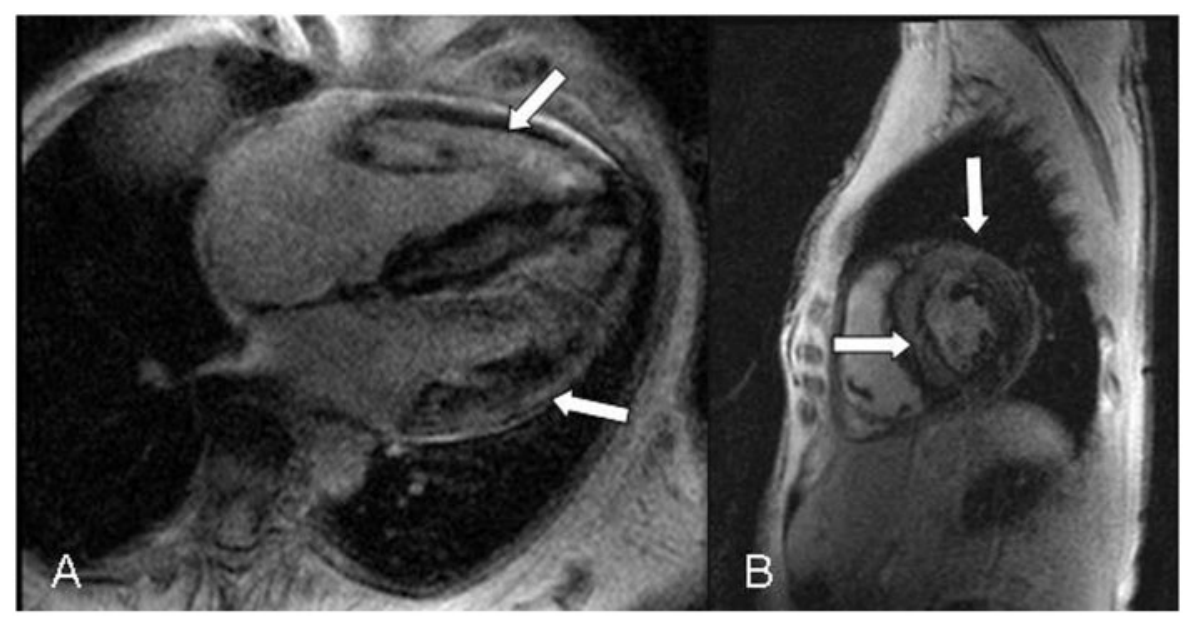

Figure 7 A 70-year-old man with a history of arrhythmia. T1-weighted gadolinium enhanced delayed imaging of the heart in 4 chamber (A), and short-axis (B) views reveal mild left and moderate right atrial enlargement, with severe diffuse thickening of the left ventricular myocardium and diffuse subendocardial late gadolinium enhancement involving all 4 chambers, consistent with biopsy-proven cardiac amyloidosis.

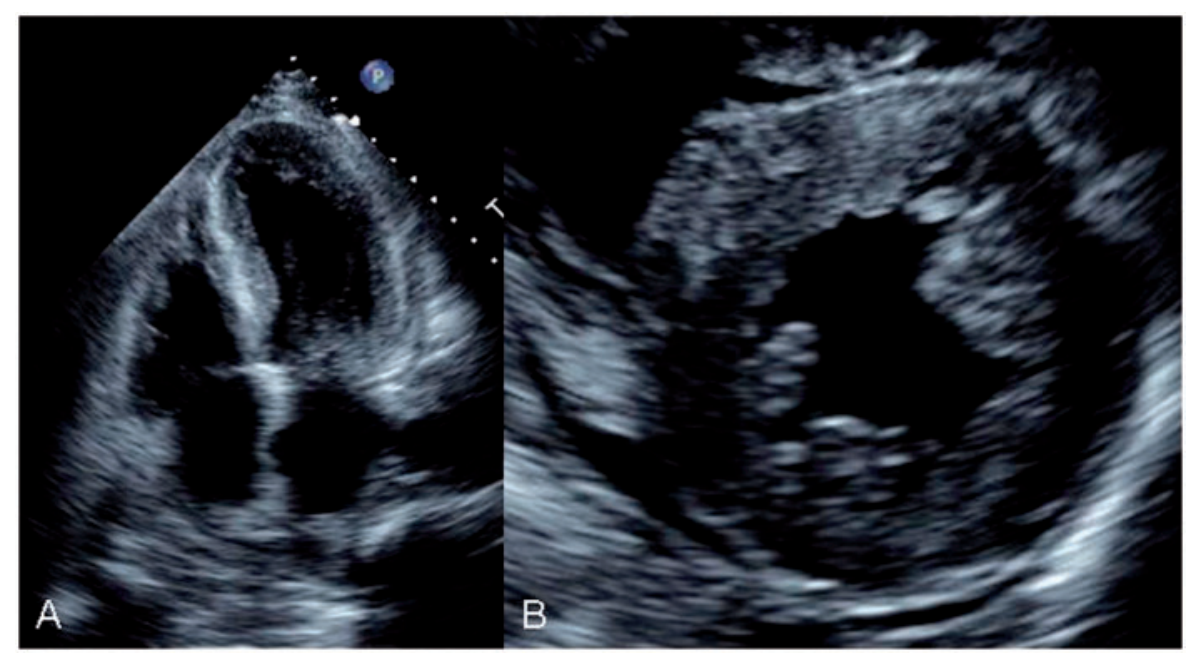

Figure 8 A 73-year-old with a history of multiple myeloma and cardiac amyloid and multiple hospital admissions for heart failure. Four-chamber (A) and short-axis (B) grayscale sonographic images reveal a small left ventricle with severe concentric left ventricular hypertrophy. Atria are borderline enlarged. Ejection fraction was preserved at $70 \%$.

without calcifications ${ }^{[12]}$. Diffuse hepatic infiltration may cause hepatomegaly ${ }^{[4]}$. Liver function tests remain normal until late in the disease, with abnormal function portending survival of less than 6 months ${ }^{[4]}$. Hepatic protein deposition appears hypoechoic on ultrasonography, and appears hyperintense on T1-weighted images, and isointense on T2-weighted images on MRI ${ }^{[12]}$. Amyloid causes splenomegaly in only $4-13 \%$ of patients with systemic amyloidosis, despite frequent involvement pathologically ${ }^{[12]}$. Splenic involvement appears hyperintense on T1-weighted images and hypointense on
T2-weighted images on MRI, with poor contrast enhancement, likely due to vascular involvement ${ }^{[12]}$. Early renal involvement causes nephromegaly (Fig. 10) with progression to small contracted kidneys with cortical thinning ${ }^{[12]}$. Sonographically diffusely infiltrated kidneys may appear abnormally echogenic, with a preserved corticomedullary junction, often with increased resistive index in the interlobar arteries ${ }^{[12]}$. Case reports describe involvement of other solid organs, either focally or diffusely, including the bladder, ovaries and adrenals, however the rare involvement of these organs precludes 


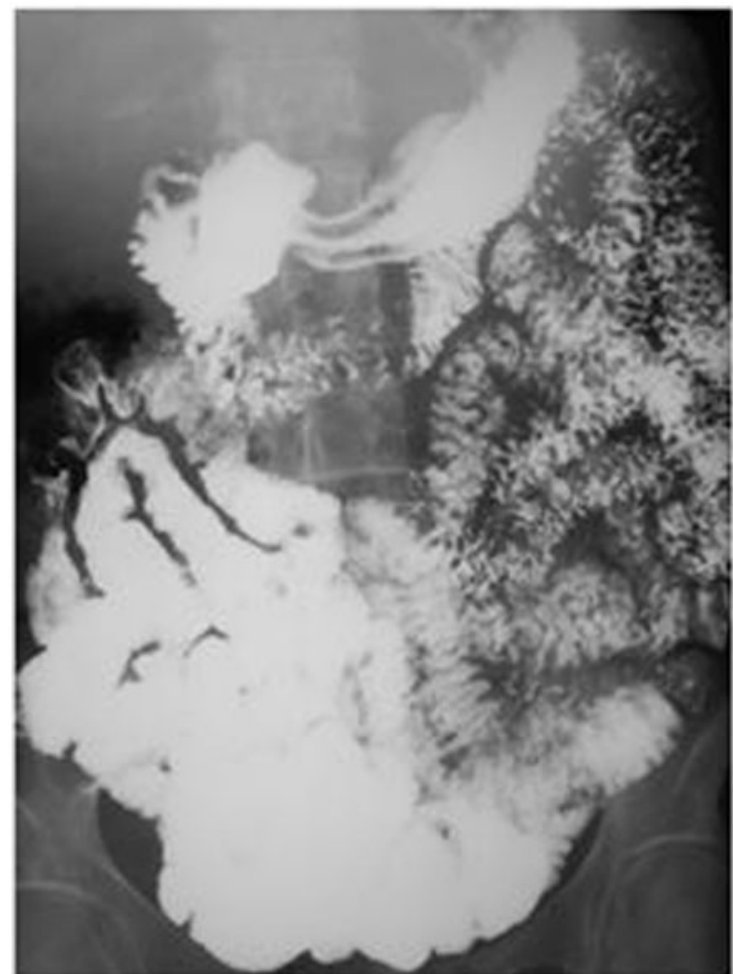

Figure 9 A 59-year-old woman presenting with abdominal pain. Fluoroscopic images obtained during a small bowel follow-through reveal diffuse, somewhat nodular fold thickening involving predominately the jejunum; biopsy yielded amyloidosis.

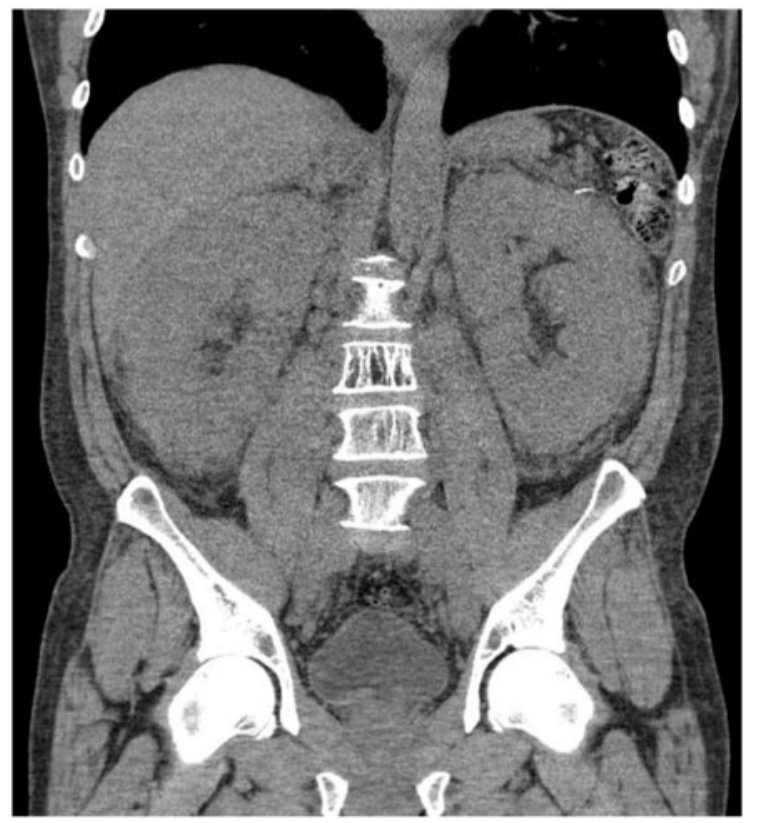

Figure 10 A 54-year-old with a history of B-cell lymphoproliferative disorder and amyloid. Non-contrast coronal CT reveals bilateral nephromegaly, with the right kidney measuring $17.8 \mathrm{~cm}$ and the left kidney measuring $17.4 \mathrm{~cm}$.

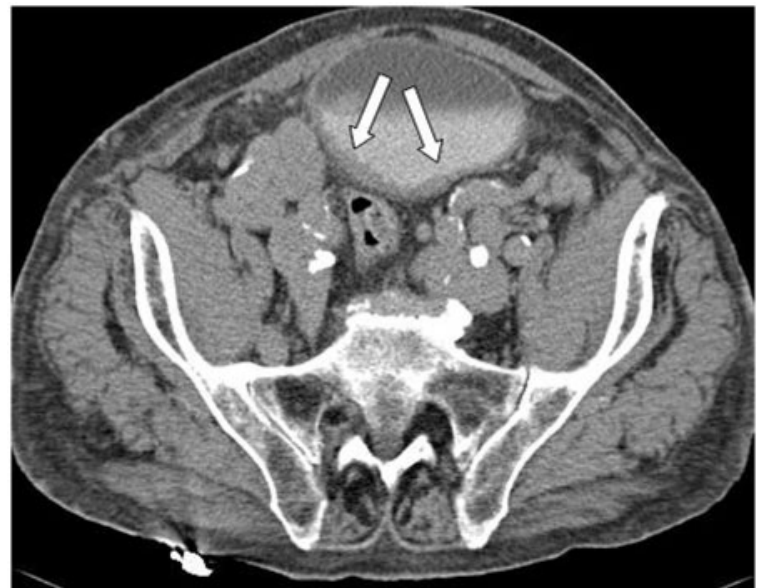

Figure 11 A 91-year-old man with urinary urgency and hematuria. Delayed images from a contrast-enhanced axial CT scan reveal diffuse thickening of the posteriolateral bladder wall. Subsequent cystoscopically guided biopsy of the posterior bladder wall revealed diffuse amyloid infiltration.

description of a "typical" appearance. Figs. 11 and 12 highlight amyloid involving the bladder and ovary.

\section{Musculoskeletal}

Musculoskeletal amyloid most frequently occurs in hemodialysis patients, manifesting as an erosive arthropathy, a rapidly destructive spondyloarthropathy, or as carpal tunnel syndrome ${ }^{[13]}$. Amyloid in the bones and joints often appears low to intermediate signal intensity on T1-/T2-weighted MR images ${ }^{[13]}$. Extremity amyloidomas are rarer than amyloidomas in other organs, and occur most frequently in the lower extremities ${ }^{[14]}$. Extremity amyloidomas occur in both primary and secondary amyloidosis, appearing as heterogeneous focal masses on both T1- and T2-weighted imaging, with variable enhancement ${ }^{[14]}$. Figs. 12, 13 and 14 highlight the varied appearance of musculoskeletal amyloid.

Given the frequent coexistence of plasma cell dyscrasias and amyloidosis, many of our patients underwent positron emission tomography (PET)/CT during their evaluation. The appearance of systemic amyloidosis on fluorodeoxyglucose (FDG)-PET/CT is variable and not specific to the disease ${ }^{[15]}$. As illustrated in Figs. 2, 3, 4 and 6, the intensity of FDG uptake on PET/CT encompasses a spectrum that ranges from mild to intense. FDG avidity in patients with concurrent amyloid and malignancy does not equate with malignant involvement. Amyloid alone may cause intense FDG avidity (Fig. 2).

\section{Conclusion}

This overview describes the clinical characteristics of primary and secondary amyloidosis, and provides examples 


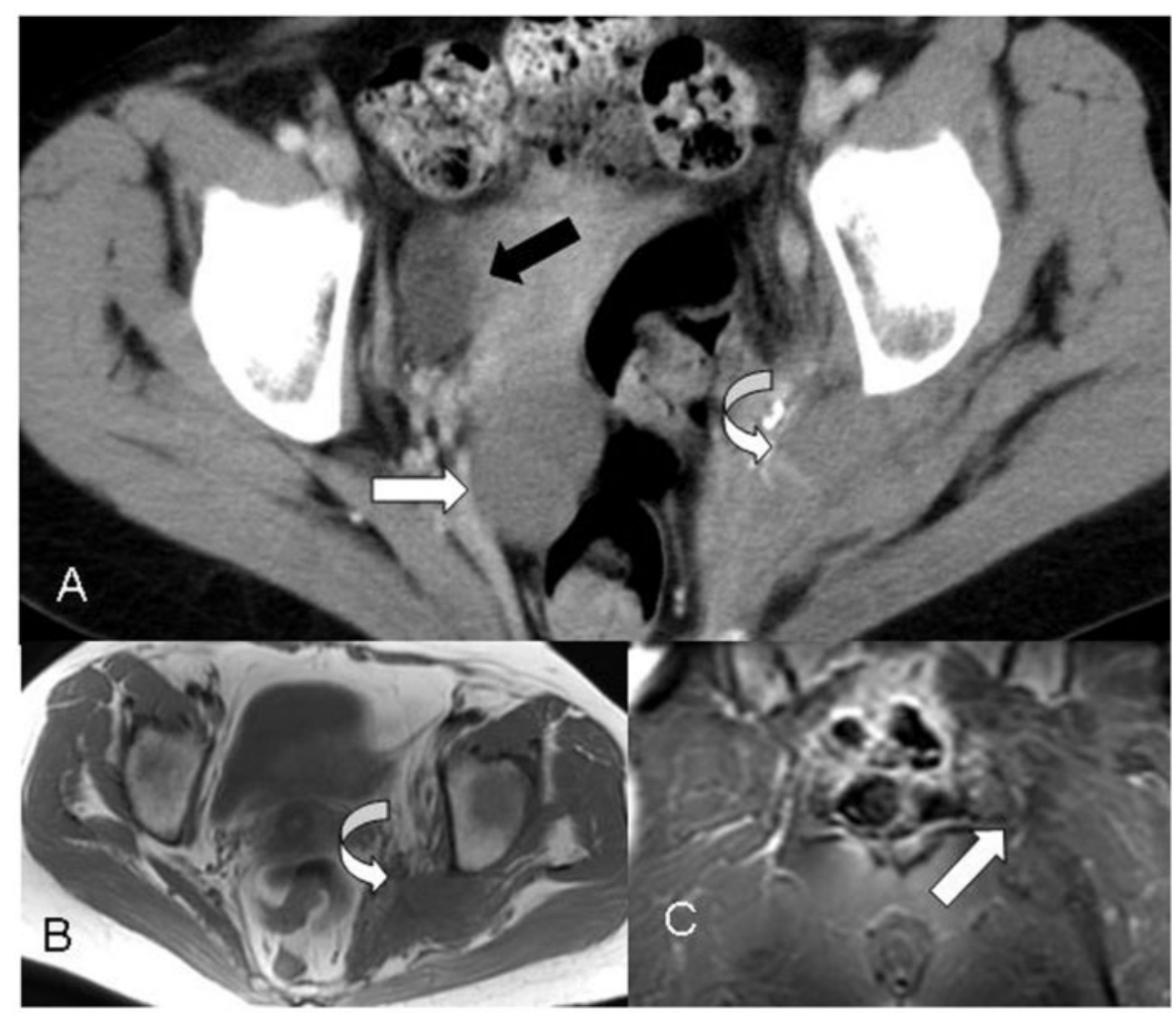

Figure 12 A 56-year-old woman with a history of lymphoplasmacytic lymphoma who presented with left buttock pain. Axial contrast-enhanced CT (A) revealed a non-enhancing, somewhat infiltrative soft tissue mass involving the left gluteal musculature (curved white arrow) extending through the sciatic foramen, with associated calcifications. Additionally, a non-enhancing lobular soft tissue mass (straight white arrow) was noted in the right adnexa. Black arrow points to a portion of a capacious bladder. Biopsy of the gluteal lesion revealed diffuse amyloid infiltration, with no evidence of lymphoma. Surgical resection of the right adnexa revealed both lymphplasmacytic lymphoma and amyloid. Axial proton density images from a subsequent MRI (B) revealed the gluteal mass to be hypointense (the adnexal lesion had been resected). Coronal T1-weighted fat-saturated image obtained after gadolinium administration (C) revealed no enhancement in this infiltrative lesion.

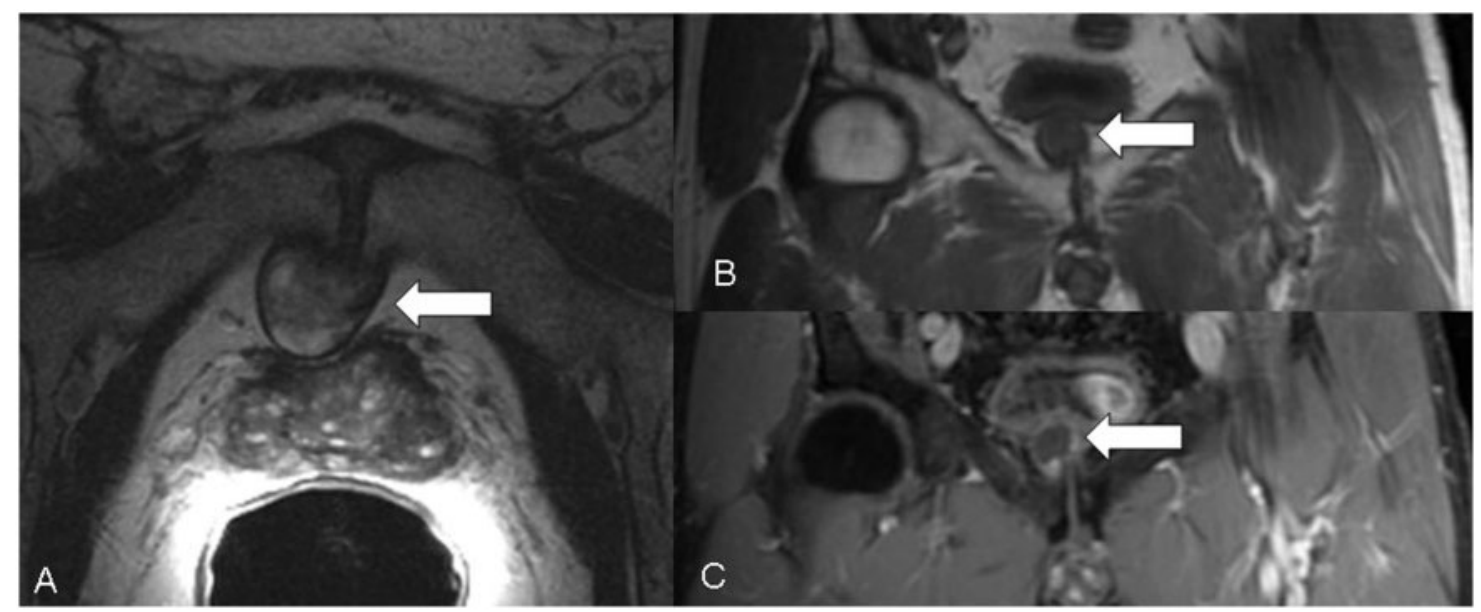

Figure 13 A 68 year-old man with a recent diagnosis of prostate cancer, found to have an incidental lesion detected on MRI. (A) T2-weighted axial MR image at the level of the pubic symphysis (with rectal coil in place posteriorly) reveals a heterogeneous, well-circumscribed extraprostatic lesion with dark rim extending from the pubic symphysis posteriorly into the pre-prostatic fat, and causing a mild mass effect on the anterior prostate. Coronal T1-weighted pre- (B), and fat-suppressed post-gadolinium images $(C)$, reveal a hypointense peripherally enhancing lesion. Pathology at surgical resection revealed a focal amyloidoma. 


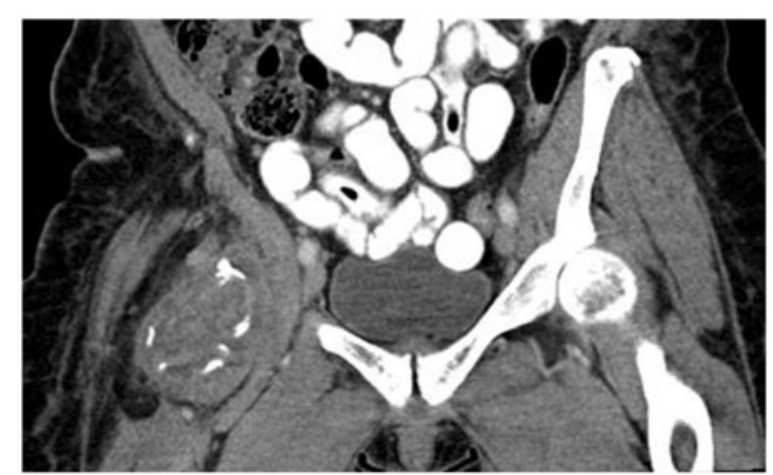

Figure 14 A 72-year-old woman with a history of multiple myeloma presenting with a right thigh mass. Coronal contrast-enhanced CT image reveals a non-enhancing heterogeneous mass in the musculature of the right hip containing chunky internal calcifications. Biopsy revealed extensive amyloid deposition.

of the varied appearances of amyloid throughout the body using multimodality imaging. Radiologists should consider amyloid in clinically perplexing chronically ill patients, particularly those with plasma cell dyscrasias or chronic inflammatory states.

\section{Acknowledgments}

We would like to thank Dr John Braver, Dr Cheryl Sadow, and Dr Hale Ersoy for their support and assistance in the preparation of this article.

\section{References}

[1] Falk RH, Comenzo RL, Skinner M. The systemic amyloidoses. N Engl J Med 1997; 337: 898-909. doi:10.1056/NEJM 199709253371306.
[2] Rajkumar SV, Dispenzieri A, Kyle RA. Monoclonal gammopathy of unknown significance, Waldenström macroglobulinemia, AL amyloidosis, and related plasma cell disorders: diagnosis and treatment. Mayo Clin Proc 2006; 81: 693-703. doi:10.4065/ 81.5.693.

[3] Urban BA, Fishman EK, Goldman SM, et al. CT evaluation of amyloidosis: spectrum of disease. Radiographics 1993; 13: $1295-1308$.

[4] Georgiades CS, Neyman EG, Barish MA, Fishman EK. Amyloidosis: review and CT manifestations. Radiographics 2004; 24: 405-416. doi:10.1148/rg.242035114.

[5] Parmar H, Rath T, Castillo M. Imaging of focal amyloid depositions in the head, neck, and spine: amyloidoma. Am J Neuroradiol 2010; 31: 1165-1170. doi:10.3174/ajnr.A1977.

[6] Yamada M. Cerebral amyloid angiopathy: an overview. Neuropathology 2000; 1: 8-22.

[7] Chao CP, Kotsenas AL, Broderick DF. Cerebral amyloid angiopathy: CT and MR imaging findings. Radiographics 2006; 26: 1517-1531. doi:10.1148/rg.265055090.

[8] Seccia V, Dallan I. A rare case of primary systemic amyloidosis of the neck with massive cervical lymph node involvement: a case report and review of the literature. Leukemia Res 2010; 24: e100-e103.

[9] Alywin AC, Gishen P, Copley S. Imaging appearance of thoracic amyloidosis. J Thorac Imaging 2005; 20: 41-46. doi:10.1097/ 01.rti.0000154074.29194.09.

[10] Gómez AT, Alonso RM, Garcia MMO, Fabbricatore AA, GarciaTejedor JL. Thoracic amyloidosis: high-resolution computed tomographic findings in 3 cases. J Comput Assist Tomogr 2008; 32: 926-928.

[11] Seeger A, Klumpp B, Kramer U, et al. MRI assessment of cardiac amyloidosis: experience of six cases with review of current literature. Br J Radiol 2009; 82: 337-342. doi:10.1259/bjr/76104551.

[12] Kim SH, Han JK, Lee KH, et al. Abdominal amyloidosis: spectrum of radiological findings. Clin Radiol 2003; 58: 610-620. doi:10.1016/S0009-9260(03)00142-9.

[13] Penrod BJ, Resnik CS. Amyloid arthropathy. Arthritis Rheum 1997; 40: 1903-1905. doi:10.1002/art.1780401028.

[14] Shim JC, Lee YW, Lee GJ, Jeon JD, Kim HK. MR finding of primary amyloid arthropathy associated with multiple myeloma. J Comput Assist Tomogr 1990; 14: 1030-1032.

[15] Glaudemans A, Slart R, Zeebregts C, et al. Nuclear imaging in cardiac amyloidosis. Eur J Nucl Med Mol Imaging 2009; 36: 702-714. doi:10.1007/s00259-008-1037-1. 